\title{
Prevalência de cárie, gengivite e fluorose em escolares de 12 anos de Porto Alegre - RS, Brasil, 1998/1999
}

\section{Prevalence of dental caries, gingivitis and fluorosis in 12-year-old schoolchildren from Porto Alegre - RS, Brazil, 1998/1999}

Berenice Barbachan e SILVA*

Marisa MALTZ**

SILVA, B. B. e; MALTZ, M. Prevalência de cárie, gengivite e fluorose em escolares de 12 anos de Porto Alegre - RS, Brasil, 1998/1999. Pesqui Odontol Bras, v. 15, n. 3, p. 208-214, jul./set. 2001.

\begin{abstract}
O objetivo do estudo foi avaliar a prevalência de cárie, gengivite e fluorose em escolares de 12 anos de Porto Alegre - RS. Foram examinados 1.000 escolares da rede de ensino público e particular da cidade. Os índices utilizados foram o ISG, $\mathrm{CPO}$ e o ITF. Após a coleta do ISG, a criança foi submetida à remoção da placa e secagem dos dentes para proceder-se aos exames de cárie e fluorose. A média e erro padrão do CPOD, incluindo as lesões não cavitadas, foi de 2,22 $\pm 0,08$, o ISG foi de $19,76 \% \pm 0,54 \%$ e a prevalência de escolares com fluorose foi de 52,9\%. Em relação à severidade, 45,9\% das crianças apresentaram ITF $1,6,1 \%$, ITF 2 e $0,9 \%$, ITF 3 . Os escolares com CPOS maior que $7(51,2 \%)$ compreenderam $14,4 \%$ da amostra. Quanto ao sangramento gengival, observou-se que $12 \%$ das crianças apresentavam ISG $\geq 40 \%$. O CPOD de Porto Alegre é comparável ao de países desenvolvidos, resultado provavelmente em decorrência do uso intensivo de flúor, que também está ocasionando a fluorose. A severidade da fluorose encontrada não justifica qualquer medida de saúde pública. As medidas de saúde pública de impacto populacional estão sendo efetivas, porém, existe uma parcela da população que necessita de cuidados adicionais.
\end{abstract}

UNITERMOS: Epidemiologia; Cárie dentária; Fluorose dentária; Gengivite; Serviços de odontologia escolar.

\section{INTRODUÇÃO}

A incidência de cárie dentária vem diminuindo em todo o mundo. A maioria dos autores atribui essa queda ao uso do flúor nas suas diversas formas. Não se observam grandes mudanças quanto à ingestão de sacarose na dieta e nem modificações acentuadas na remoção de placa ${ }^{8,13}$. Essa associação de formas de uso de flúor tem sido apontada, também, como causa do aumento na incidência de fluorose. Porto Alegre é uma cidade com água de abastecimento fluoretada artificialmente desde 1975. A partir do final dos anos 80 , a maioria dos dentifrícios, inclusive os de preço mais acessivel, passaram a ser fluoretados. Acredita-se que essa medida provocou impacto populacional, tanto na incidência de cárie, como na incidência de fluorose $^{10}$.

O objetivo do presente estudo foi avaliar a prevalência e severidade de cárie, gengivite e fluorose em escolares de 12 anos de Porto Alegre, RS, Brasil.

\section{MATERIAL E MÉTODOS}

Este levantamento foi realizado nos anos de 1998 e 1999, na cidade de Porto Alegre, que conta com flúor adicionado na água de abastecimento público em uma concentração de $0,7 \mathrm{ppm}$ a $1 \mathrm{ppm}$. O trabalho foi aprovado pelo Comitê de Ética da UFRGS.

A amostra representativa constou de 1.000 escolares, com idade média de 12 anos e 5 meses, residentes desde o nascimento na cidade $(44,7 \%$ sexo masculino; 55,3\% - sexo feminino). Foram examinadas crianças provenientes de um total de 36 escolas, $27,4 \%$ da rede particular e $72,6 \%$ da rede pública.

Os responsáveis responderam a um questionário sobre: renda familiar, acesso a diferentes compostos fluoretados, tratamento odontológico e cuidados com higiene bucal. Em 375 questionários, foi acrescentada uma pergunta sobre possiveis queixas a respeito dos dentes dos escolares para mensurar-se a percepção dos pais em relação às manchas decorrentes de fluorose.

*Professora Assistente; **Professora Titular - Faculdade de Odontologia da Universidade Federal do Rio Grande do Sul. 
SILVA, B. B. e; MALTZ, M. Prevalência de cárie, gengivite e fluorose em escolares de 12 anos de Porto Alegre - RS, Brasil, $1998 / 1999$.

Pesqui Odontol Bras, v. 15, n. 3, p. 208-214, jul./set. 2001.

O exame foi realizado na própria escola, com a utilização de foco de luz, compressor a ar, odontoscópio, sonda exploradora e sonda periodontal. A sistemática adotada para exame foi: (1) determinação do Índice de Sangramento Gengival (ISG) ${ }^{20}$; (2) remoção profissional da placa com escova, dentifrício e fio dental; (3) secagem dos dentes por 30 segundos; (4) determinação do Índice de Superficies Cariadas Perdidas e Obturadas (CPOS) ${ }^{6}$; (5) determinação do Índice de Fluorose de Thylstrup e Fejerskov $(\mathrm{ITF})^{19}$.

No índice CPO, foi analisada a presença de lesões não cavitadas, cavitadas, superficies restauradas (R) ou perdidas (EI - extração indicada; E extraído). As lesões foram classificadas segundo os seguintes critérios: (1) lesão não cavitada ativa (LNC) - aparência clínica de esmalte opaco e esbranquiçado; (2) lesão cavitada ativa - destruição da superfície com esmalte opaco e dentina amolecida de coloração clara; (3) lesão cavitada inativa destruição da superfície com esmalte brilhante, dentina endurecida e escurecida. As cavidades foram classificadas quanto à necessidade de tratamento restaurador (LCAR e LCIR). As lesões cavitadas ativas pequenas, com pouco envolvimento dentinário e as lesões cavitadas inativas que não acumulavam placa e não tinham comprometimento pulpar e/ou funcional foram consideradas como sem necessidade restauradora (LCA e LCI, respectivamente). As superficies seladas foram consideradas hígidas.

A determinação dos índices CPO, do ISG e do ITF foi realizada por um cirurgião-dentista. O examinador procedeu a exames duplos em dois mo- mentos do estudo, com o objetivo de determinar sua reprodutibilidade - antes de iniciar o levantamento e na última escola examinada. Na primeira avaliação, foram reexaminadas 50 crianças, a concordância no CPOS foi de $99 \%$ e o índice kappa foi 0,83 e, no ITF, a concordância foi de $91 \%$ e índice kappa foi 0,68. Na etapa final, foram reexaminadas 11 crianças, obtendo-se, no CPOS, uma concordância de 98\% e índice kappa de 0,80 e, no ITF, $92 \%$ e 0,60 , respectivamente. Os resultados demonstram um alto nível de reprodutibilidade em relação ao CPOS e um bom ajuste no ITF.

Para análise dos dados, foram realizados testes $t$ de Student (SPSS para Windows). As médias dos eventos coletados encontram-se sempre acompanhadas do respectivo erro padrão.

\section{RESULTADOS}

Neste estudo, 52,4\% das famílias responderam possuir uma renda per capita de até 2 salários mínimos e $11,1 \%$ acima de 5 salários mínimos. Este dado foi omitido em $20,5 \%$ dos questionários. Em relação ao tipo de tratamento odontológico recebido, $55 \%$ relataram buscar atendimento em clínicas privadas.

Não foi observada diferença estatística entre os sexos em nenhum dos eventos examinados.

$\mathrm{O}$ índice CPO foi calculado incluindo e não incluindo as LNC, tendo como objetivo possibilitar comparações com estudos que não incluem este estágio da lesão em seus levantamentos. A média e erro padrão do CPOS sem inclusão das LNC foi de $2,58 \pm 0,13$ e com estas lesões, de 3,22 $\pm 0,14$ e o

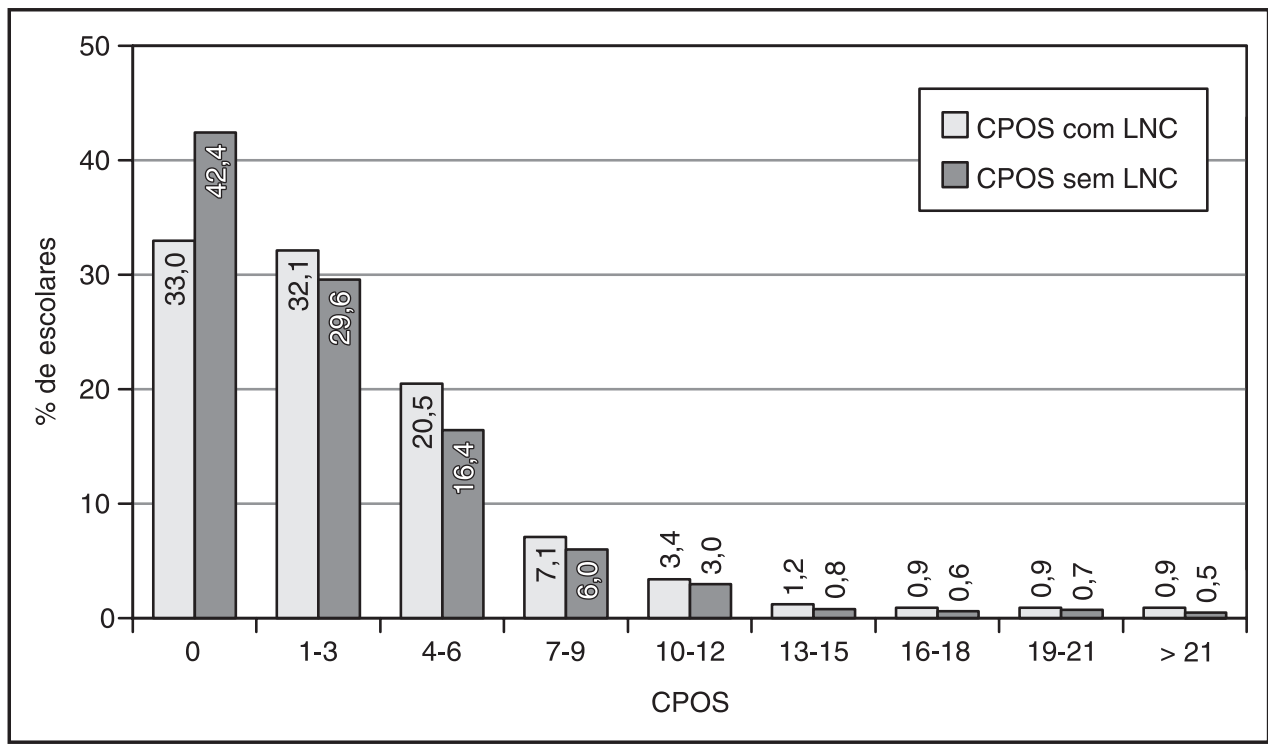

GRÁFICO 1 - Distribuição do percentual dos escolares de 12 anos de Porto Alegre em relação ao CPOS. Porto Alegre RS, 1999. LNC = lesão não cavitada ativa. 
SILVA, B. B. e; MALTZ, M. Prevalência de cárie, gengivite e fluorose em escolares de 12 anos de Porto Alegre - RS, Brasil, $1998 / 1999$.

Pesqui Odontol Bras, v. 15, n. 3, p. 208-214, jul./set. 2001.

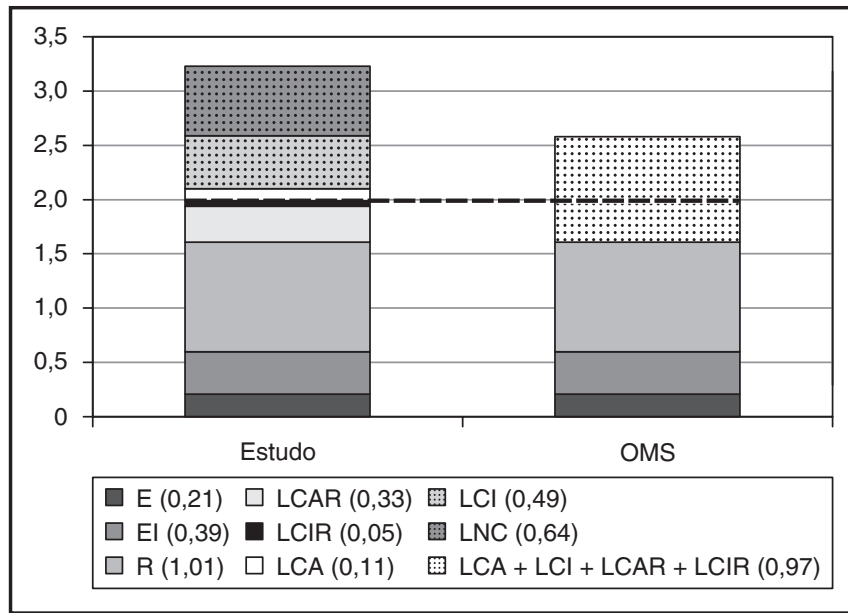

GRÁFICO 2 - Composição da média do CPOS nos escolares de 12 anos segundo critérios preconizados pelo estudo e pela OMS. Porto Alegre - RS, 1999. E = extraído; $\mathrm{EI}=$ extração indicada; $\mathrm{R}=$ restaurada LCAR = lesão cavitada ativa com indicação de restauração; LCIR = lesão cavitada inativa com indicação de restauração; LCA = lesão cavitada ativa; $\mathrm{LCI}=$ lesão cavitada inativa; $\mathrm{LNC}=1 \mathrm{e}$ são não cavitada ativa. Acima da linha pontilhada estão as lesões sem necessidade de tratamento restaurador.

CPOD foi de $1,63 \pm 0,06$ e 2,22 $\pm 0,08$, respectivamente.

A maioria da população estudada $(85,6 \%)$ apresenta um CPOS (incluindo as LNC) igual ou menor do que 6 (Gráfico 1). Quando analisamos a experiência de cárie no grupo com $\mathrm{CPOS} \leq 6$, observamos que ela é semelhante $(48,8 \%)$ à do grupo com CPOS maior do que $7(51,2 \%)$ que compreende somente $14,4 \%$ dos escolares.

O Gráfico 2 mostra a composição da média do indice CPOS em duas modalidades: a da OMS e a preconizada pelo estudo. Os componentes predominantes são as superfícies cavitadas $(0,97 \pm 0,06)$ e as restauradas $(1,01 \pm 0,06)$. Apesar de o CPOS ser baixo, há superfícies perdidas por cárie $(\mathrm{EI}=0,39 \pm 0,07 ; \mathrm{E}=0,21 \pm 0,04)$. No presente estudo, as cavidades de cárie foram classificadas em LCA $(0,44 \pm 0,04)$ e LCI $(0,54 \pm 0,04)$ e também quanto à indicação de restauração. Dentre as superficies que apresentaram cavidades ativas, $67,98 \%$ necessitavam de restauração, as outras eram passiveis de controle. Das LCI, apenas 7,95\% tinham indicação de restauração. A linha pontilhada no Gráfico 2 indica a separação entre as cavidades com e sem necessidade de tratamento restaurador. Em média, a necessidade de tratamento (cavidades com indicação de restauração e extra-

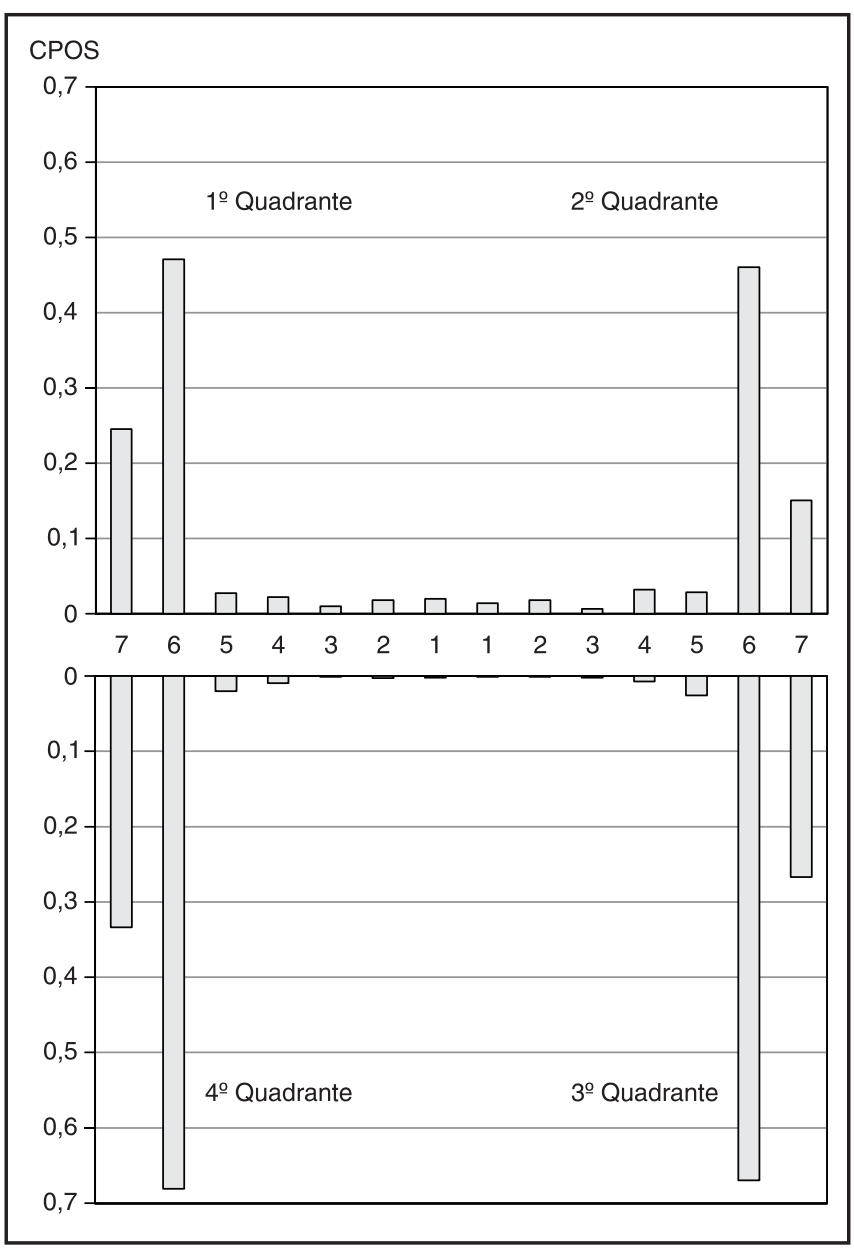

GRÁFICO 3 - Distribuição do CPOS dos escolares de 12 anos em relação ao dente atingido. Porto Alegre - RS, 1999.

ções indicadas) foi de $0,77 \pm 0,09$ superfície e de tratamento executado foi de 1,22 $\pm 0,08$ superficie (restaurada ou extraída por cárie).

Os dentes mais afetados por cárie foram os primeiros molares, seguidos pelos segundos (Gráfico 3). A arcada inferior apresentou uma experiência de cárie significativamente maior do que a arcada superior $(\mathrm{p}<0,05)$. As superficies dentárias mais atingidas foram as oclusais $(1,90 \pm 0,07)$, seguidas pelas livres $(0,93 \pm 0,05)$ e proximais $(0,38 \pm$ 0,04).

A Tabela 1 mostra a composição do CPOS na superfície oclusal do primeiro e do segundo molar. Observa-se no primeiro molar um predomínio de superficies restauradas, enquanto, no segundo, há um predomínio de LNC. A Tabela 2 mostra a experiência de lesão de cárie ativa, e a Tabela 3, a experiência de lesão de cárie inativa. Observa-se, no 
SILVA, B. B. e; MALTZ, M. Prevalência de cárie, gengivite e fluorose em escolares de 12 anos de Porto Alegre - RS, Brasil, $1998 / 1999$.

Pesqui Odontol Bras, v. 15, n. 3, p. 208-214, jul./set. 2001.

TABELA 1 - Número (n) e percentagem (\%) da experiência de cárie da superfície oclusal do primeiro e segundo molares permanentes $\left(1^{\circ} \mathrm{MP}\right.$ e $\left.2^{\circ} \mathrm{MP}\right)$ dos escolares de 12 anos. Porto Alegre - RS, 1999.

\begin{tabular}{l|r|r|r|r}
\hline \hline \multirow{2}{*}{ Superfície Oclusal } & \multicolumn{2}{|c|}{$1^{\circ} \mathrm{MP}$} & \multicolumn{2}{c}{$2^{\circ} \mathrm{MP}$} \\
\cline { 2 - 5 } & \multicolumn{1}{c|}{$\mathrm{n}$} & \multicolumn{1}{c|}{$\%$} & \multicolumn{1}{c}{$\mathrm{n}$} & \multicolumn{1}{c}{$\%$} \\
\hline Hígida & 2.790 & 69,9 & 2.161 & 78,8 \\
\hline Lesão não cavitada & 36 & 0,9 & 464 & 16,9 \\
\hline Lesão cavitada & 443 & 11,1 & 83 & 3,0 \\
\hline Restaurada & 612 & 15,3 & 34 & 1,2 \\
\hline Extração indicada & 73 & 1,8 & 2 & 0,1 \\
\hline Total & 3.954 & 100 & 2.744 & 100 \\
\hline \hline
\end{tabular}

TABELA 2 - Número (n) e percentagem (\%) da experiência de lesão cavitada ativa do primeiro e segundo molares permanentes $\left(1^{\circ} \mathrm{MP}\right.$ e $\left.2^{\circ} \mathrm{MP}\right)$ dos escolares de 12 anos. Porto Alegre - RS, 1999.

\begin{tabular}{l|c|c|c|c}
\hline \hline \multirow{2}{*}{ Lesão cavitada ativa } & \multicolumn{2}{|c|}{$1^{\circ} \mathrm{MP}$} & \multicolumn{2}{c}{$2^{\circ} \mathrm{MP}$} \\
\cline { 2 - 5 } & $\mathrm{n}$ & $\%$ & $\mathrm{n}$ & $\%$ \\
\hline $\begin{array}{l}\text { Lesão cavitada ativa que } \\
\text { não requer restauração }\end{array}$ & 45 & 1,1 & 19 & 0,7 \\
\hline $\begin{array}{l}\text { Lesão cavitada ativa que } \\
\text { requer restauração }\end{array}$ & 122 & 3,1 & 22 & 0,8 \\
\hline Total & 167 & 4,2 & 41 & 1,5 \\
\hline \hline
\end{tabular}

primeiro molar, um grande número de LCI sem necessidade de tratamento restaurador.

Do total de examinados, 97,4\% apresentaram sangramento gengival com uma grande variação na amostra $(19,76 \% \pm 0,54 \%)$. O ISG por criança não foi alto, 35,3\% das crianças apresentaram ISG de $10 \%$ ou menos, apenas $12 \%$ das crianças apresentaram ISG $\geq 40 \%$.

A fluorose foi detectada em $52,9 \%$ das crianças, sendo que $47,1 \%$ estavam livres de fluorose, $45,9 \%$ apresentaram ITF $1,6,1 \%$, ITF 2 e $0,9 \%$, ITF 3. O Gráfico 4 mostra a fluorose quanto ao dente atacado, classificando a sua severidade segundo o grau do ITF. Os dentes mais atingidos foram os segundos molares, seguidos pelos pré-molares.

Em relação à escovação dentária, $84,6 \%$ responderam escovar seus dentes de 2 a 3 vezes por dia. Somente $19,85 \%$ da amostra utilizava fio dental regularmente. Quase a totalidade da amostra respondeu utilizar dentifrício fluoretado $(99,6 \%)$. Aplicações profissionais de flúor foram recebidas
TABELA 3 - Número (n) e percentagem (\%) da experiência de lesão cavitada inativa do primeiro e segundo molares permanentes ( $1^{\circ} \mathrm{MP}$ e $\left.2^{\circ} \mathrm{MP}\right)$ dos escolares de 12 anos. Porto Alegre - RS, 1999.

\begin{tabular}{l|c|c|c|c}
\hline \hline \multirow{2}{*}{ Lesão cavitada inativa } & \multicolumn{2}{|c|}{$1^{\circ} \mathrm{MP}$} & \multicolumn{2}{c}{$2^{\circ} \mathrm{MP}$} \\
\cline { 2 - 5 } & $\mathrm{n}$ & $\%$ & $\mathrm{n}$ & $\%$ \\
\hline $\begin{array}{l}\text { Lesão cavitada inativa que } \\
\text { não requer restauração }\end{array}$ & 255 & 6,4 & 41 & 1,5 \\
\hline $\begin{array}{l}\text { Lesão cavitada inativa que } \\
\text { requer restauração }\end{array}$ & 21 & 0,5 & 1 & 0,0 \\
\hline Total & 276 & 6,9 & 42 & 1,5 \\
\hline \hline
\end{tabular}

por $76,4 \%$ da amostra. Solução de flúor para bochecho foi utilizada, em algum momento, por $71,2 \%$.

Dos 3 casos de queixas dos pais relacionadas a manchas nos dentes, apenas uma criança apresentava fluorose - ITF 2 - em dentes anteriores.

\section{DISCUSSÃO/CONCLUSÕES}

A escolha da idade de 12 anos foi feita por ser esta uma idade freqüentemente utilizada em levantamentos epidemiológicos de cárie. FEJERSKOV et $a .^{5}$ (1992) consideram a idade de 12 anos ideal para examinar-se fluorose, pois, nessa idade, pode-se analisar dentes recém-erupcionados que, por ter maturação do esmalte prolongada, são mais suscetíveis. Nessa idade, a evasão escolar ainda não é acentuada.

O CPOD aos 12 anos em Porto Alegre é baixo, semelhante ao de outras cidades brasileiras ${ }^{1,15,21} \mathrm{e}$ comparável ao de países industrializados.

Aumentou-se em cerca de $20 \%$ a experiência de cárie, ao incluírem-se as LNC. É discutível a inclusão do exame das LNC nos levantamentos. Um dos fatores que justificam a não-inclusão deste estágio da lesão é a possivel perda da confiabilidade dos dados. Entretanto, o índice kappa, no presente estudo, demonstrou um alto grau de reprodutibilida$\mathrm{de}^{12}$. Esta não-inclusão causa uma subestimação da doença. $\operatorname{ISMAIL}^{9}$ (1997) afirma que as LNC são as mais prevalentes e determinam uma redução de $1 / 4$ na proporção de indivíduos livres de cárie. No presente estudo, esta proporção caiu de 42,4\% para 33,0\% quando incluíram-se as LNC (Gráfico 1$)$. As LNC e as LCA $(32,02 \%$ das lesões cavitadas ativas) podem ser paralisadas por intervenções não operatórias simples, sendo, portanto, necessária a coleta destes dados em levantamentos epidemiológicos. 
SILVA, B. B. e; MALTZ, M. Prevalência de cárie, gengivite e fluorose em escolares de 12 anos de Porto Alegre - RS, Brasil, $1998 / 1999$.

Pesqui Odontol Bras, v. 15, n. 3, p. 208-214, jul./set. 2001.

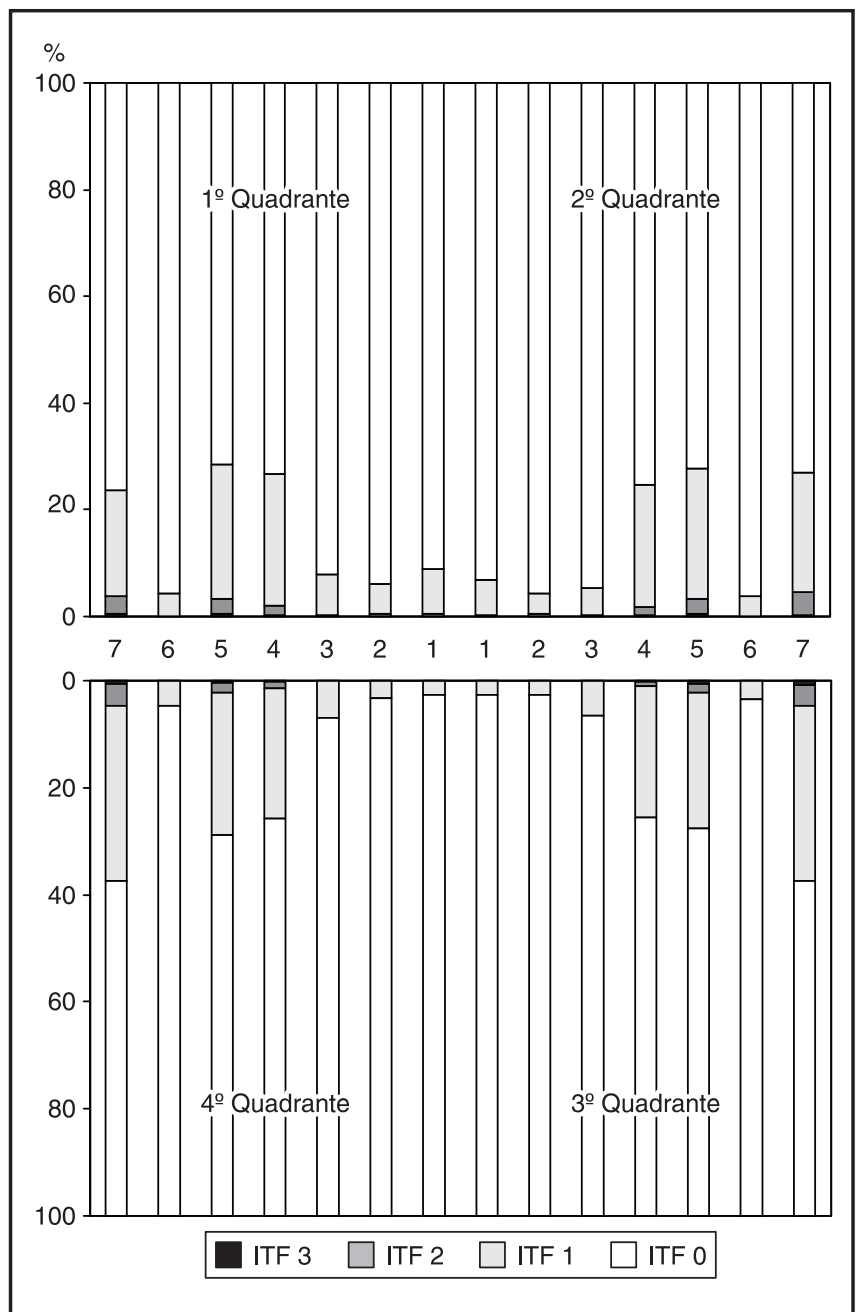

GRÁFICO 4 - Distribuição percentual do Índice de Fluorose de Thylstrup e Fejerskov em relação aos dentes atingidos e ao grau de severidade nos escolares de 12 anos. Porto Alegre - RS, 1999.

O Gráfico 2 mostra a média do CPOS subdividida em todos os componentes utilizados no estudo e segundo os critérios da OMS. A OMS, por um lado, subestima a doença ao não incluir as lesões iniciais e, por outro, a superestima, indicando tratamento restaurador onde este não seria necessário. A linha pontilhada separa a não necessidade de intervenção restauradora nas lesões, reconhecida no sistema de diagnóstico utilizado no estudo. Acima desta linha, quando projetada sobre a coluna correspondente aos critérios da OMS, observa-se a indicativa de tratamento restaurador desnecessária que seria motivada, uma vez que a toda superficie cavitada estaria preconizada uma restauração. É importante salientar que 92,05\% das lesões inativas que não necessitam ser restau- radas representam história passada de cárie, não podendo ser incluídas no cálculo que irá planejar o tratamento desta população. O decréscimo da prevalência de cárie na maior parte do mundo exige um critério de diagnóstico mais sensivel. O exame deve permitir a identificação das diversas instâncias da doença, para que se possa atuar prontamente e para que o critério indicativo de restauração seja correto9.

Um grande número de lesões de cárie concentrou-se em poucos indivíduos (Gráfico 1). Somente $14,4 \%$ da amostra é responsável por $51,2 \%$ da experiência de cárie, enquanto $48,8 \%$ do restante da experiência de cárie corresponde a mais de $80 \%$ da amostra. Esta informação é importante para o planejamento em saúde pública, possibilitando uma alocação de recursos direcionada à parcela da população mais necessitada ${ }^{16}$.

Os primeiros molares, seguidos pelos segundos, foram os dentes mais atingidos pela cárie havendo uma maior prevalência na arcada inferior (Gráfico 3), confirmando dados de diversos levantamentos epidemiológicos ${ }^{11,12,14,17,22}$. As lesões concentram-se nas superficies oclusais. Nos primeiros molares, $69,9 \%$ das superficies oclusais eram hígidas, as outras apresentam, na sua maioria, história passada de doença. A atividade cariogênica, neste situ, inicia-se no período de erupção. Neste período, ocorre uma retenção maior de biofilme dentário, sendo sua remoção dificultada, prolongando-se esta situação até os dentes entrarem em oclusão, ao redor dos 8 anos de idade, quando o acúmulo de placa torna-se menor ${ }^{2}$. Isto justifica o fato da grande ocorrência de pequenas cavidades inativas e a quase inexistência de lesões não cavitadas ativas, na idade de 12 anos. Na superfície oclusal do segundo molar, ocorre este mesmo processo. No momento do exame, observou-se um grande número de lesões não cavitadas ativas, provavelmente devido ao fato de, nesta idade, estes não estarem completamente erupcionados (Tabela 1). KUZMINA; EKSTRAND ${ }^{11}$ observaram que $70 \%$ da experiência de cárie das crianças de 12 anos concentrou-se no primeiro molar, sendo o segundo molar o segundo dente mais atingido. Os autores discutem que o longo período de erupção que caracteriza os molares faz com que estes sejam mais sujeitos ao ataque cariogênico. Isso não acontece com os pré-molares, que, apesar de terem a superficie oclusal com a anatomia semelhante aos molares, têm um curto periodo de erup- 
SILVA, B. B. e; MALTZ, M. Prevalência de cárie, gengivite e fluorose em escolares de 12 anos de Porto Alegre - RS, Brasil, $1998 / 1999$.

Pesqui Odontol Bras, v. 15, n. 3, p. 208-214, jul./set. 2001.

ção. Este fato pode explicar a baixa prevalência de cárie observada nos pré-molares (Gráfico 3).

$\mathrm{O}$ sangramento gengival foi bastante prevalente, estando presente na quase totalidade das crianças examinadas $(97,4 \%)$, o que está de acordo com outros estudos ${ }^{11}$. O ISG por criança, porém, não foi alto: $35,3 \%$ das crianças apresentaram ISG $\leq 10 \%$. A média do ISG encontrada foi de $19,76 \%$, havendo grande variabilidade. É essencial a inclusão da coleta do ISG nos estudos epidemiológicos, objetivando a incorporação de medidas relacionadas ao seu controle.

Observa-se um aumento na prevalência de fluorose na última década ${ }^{3,4,7,10}$. Em Porto Alegre, entre 1987 e 1997, a prevalência de fluorose em crianças de $8-9$ anos de idade passou de $7,7 \%$ para $33 \%{ }^{18}$. O presente estudo mostrou alta prevalência de crianças com este agravo (52,9\%). Entretanto, a severidade foi baixa: $93 \%$ dos examinados apresentam ITF 0 e ITF 1 e somente $7 \%$ tem ITF 2 e ITF 3 . Os dentes mais atingidos foram os pré-molares e os segundos molares ${ }^{20}$, provavelmente, por estes dentes apresentarem um período de maturação mais longo, ficando expostos ao risco da fluorose por um período maior de tempo. O grau de severidade da fluorose encontrada não significa problema de saúde pública, mesmo a pequena prevalência de ITF 3 não é percebida pela maioria das pessoas. Como a fluorose, salvo nos casos mais graves, é um problema cosmético, quando o paciente não a percebe, qualquer tratamento torna-se desnecessário.

O presente estudo demonstrou que os escolares de 12 anos de Porto Alegre têm a experiência de cárie baixa. Mais da metade desta experiência concentra-se em $14,4 \%$ da amostra. A mesma polarização é observada em relação ao sangramento gengival. Esta pequena parcela de indivíduos necessita de tratamento diferenciado para o controle destas doenças. O restante da população deve ter acesso a medidas de impacto populacional. Em relação à fluorose, observa-se que o somatório do uso de compostos fluoretados não tem tornado este agravo um problema de saúde pública.

\section{AGRADECIMENTOS}

Aos alunos de iniciação científica da UFRGS: Alessandra da Silva, Adriane Afonso, Karina Bernhard e Pablo Wüst, ao estatístico Sérgio Kato, à Dra. Mariza Favarini, Coordenadora da Política de Saúde Bucal da Secretaria Municipal de Saúde de Porto Alegre.

SILVA, B. B. e; MALTZ, M. Prevalence of dental caries, gingivitis and fluorosis in 12-year-old schoolchildren from Porto Alegre - RS, Brazil, 1998/1999. Pesqui Odontol Bras, v. 15, n. 3, p. 208-214, jul./set. 2001.

The aim of the study was to evaluate the prevalence of dental caries, gingival bleeding and fluorosis in twelve-year-old schoolchildren from Porto Alegre, Brazil. One thousand students from private and public schools were examined. The indexes used were BI, DMF and TFI. The data pertaining to BI were the first to be collected. After that, the children were submitted to plaque removal and their teeth were dried prior to the examination for caries and fluorosis. The average value for DMFT, including cavitated and non-cavitated lesions, was $2.22 \pm 0.08$ (standard error). The average value for $\mathrm{BI}$ was $19.76 \% \pm 0.54 \%$ (standard error) and the prevalence of students with fluorosis was $52.9 \%$. Regarding the severity of fluorosis, $45.9 \%$ of the children had TFI 1, $6.1 \%$ TFI 2 and $0.9 \%$ TFI 3 . A polarization of the experience of caries and gingivitis was observed in the studied population. Only $14.4 \%$ of the students had DMFS higher than 7 . A small percentage of children (12\%) presented with $\mathrm{BI} \geq 40 \%$. The experience of caries and gingivitis of 12 -year-old schoolchildren in Porto Alegre is comparable to that of children from developed countries. That probably results from the intense utilization of fluoride, which is also causing an increased prevalence of fluorosis. The severity of fluorosis does not justify any public health measure. The results show that the employed public health measures are effective, although part of the population still needs additional care.

UNITERMS: Epidemiology; Dental caries; Dental fluorosis; Gingivitis; School dentistry.

\section{REFERÊNCIAS BIBLIOGRÁFICAS}

1. BRASIL. Ministério da Saúde. Secretaria Executiva. SUS. Datasus. Levantamento epidemiológico em saúde bucal. Cárie dental. Capitais. [Online]. 1996. Disponivel: http://www.datasus.gov.br/cgi/tabcgi.exe?sbucal/sbbr.def. 15 set 1999.

2. CARVALHO, J. C.; EKSTRAND, K. R.; THYLSTRUP, A. Dental plaque and caries on occlusal surfaces of first per- manent molars in relation to stage of eruption. J Dent Res, v. 68, n. 5, p. 773-779, May 1989.

3. CORTES, D. F.; ELLWOOD, R. P.; O'MULLANE, D. M. et al. Drinking water fluoride levels, dental fluorosis and caries experience in Brazil. J Public Health, v. 56, n. 4, p. 226-228, Summer 1996.

4. ELY, H. C. Fluorose e cárie dentária em cidades do RS: estudo epidemiológico em locais com diferentes niveis de flúor nas águas. Porto Alegre, 1999. 218 p. Dis- 
SILVA, B. B. e; MALTZ, M. Prevalência de cárie, gengivite e fluorose em escolares de 12 anos de Porto Alegre - RS, Brasil, $1998 / 1999$.

Pesqui Odontol Bras, v. 15, n. 3, p. 208-214, jul./set. 2001.

sertação [Mestrado] - Faculdade de Odontologia, Pontifícia Universidade Católica.

5. FEJERSKOV, O.; FIROZE, M.; VIBEKE, B. Fluorose dentária - um manual para profissionais da Saúde. São Paulo, 1992. 122 p.

6. GUSTAFSSON, B. E.; QUENSEL, C. E.; LANKE, L. K. S. et al. The Vipeholm dental caries study. The effect of different levels of carbohydrate intake on caries activity in 436 individuals observed for five years (Sweden). Acta Odontol Scand, v. 11, p. 232-264, 1954.

7. HEINTZE, S. D.; BASTOS, J. R. M. Urinary fluoride levels and prevalence of dental fluorosis in three Brazilian cities with different fluoride concentration in drinking water. Community Dent Oral Epidemiol, v. 26, n. 5, p. 316-323, Oct. 1998.

8. IRIGOYEN, M. E.; SÁNCHEZ-HINOJOSA, G. Changes in dental caries prevalence in 12-year-old students in the state of Mexico after 9 years of salt fluoridation. Caries Res, v. 34, n. 4, p. 303-307, July/Aug. 2000.

9. ISMAIL, A. I. Clinical diagnosis of precavitated carious lesions. Community Dent Oral Epidemiol, v. 25, p. 13-23, 1997.

10. JACKSON, R.; KELLY, S.; BRIZENDINE, E. et al. Prevalence of dental fluorosis - a 33-month follow-up. J Dent Res, v. 75 (Special Issue), p. 323, 1996. [Abstract 3,313]

11. KUZMINA, I. N.; EKSTRAND, K. R. Dental caries among children from Solntsevsky - a district in Moscow, 1993. Community Dent Oral Epidemiol, v. 23, n. 5, p. 266-270, Oct. 1995.

12. MACHIULSKIENE, V.; NYVAD, B.; BAELUM, V. Prevalence and severity of dental caries in 12-year-old children in Kaunas, Lithuania, 1995. Caries Res, v. 32, n. 3, p. 175-180, May/June 1998.

13. OPPERMANN, R. V.; RÖSING, C. K. Prevenção e tratamento das doenças periodontais. In: ABOPREV. Promo- ção de saúde bucal. São Paulo : Artes Médicas, 1997. p. 255-282.

14. OKAWA, Y.; TOKAHASHI, Y.; SUZUKA, J. et al. Decline in caries prevalence in 6-14-year-old schoolchildren during 1975-85 in Shizuoka, Japan. Community Dent Oral Epidemiol, v. 20, n. 5, p. 246-249, Oct. 1992.

15. PINTO, V. G. Estudo epidemiológico sobre a prevalência da cárie dental em crianças de 3 a 14 anos. Porto Alegre : SESI - DN, 1996. 52 p.

16. ROSE, G. The strategy of preventive medicine. 4. ed. New York : Oxford University Press, 1995. 138 p.

17. SHEIHAM, A. Impact of dental treatment on the incidence of dental caries in children and adults. Community Dent Oral Epidemiol, v. 25, n. 1, p. 104-112, Feb. 1997.

18. SILVA, B. B.; MALTZ, M.; SCHAEFFER, A. Prevalence of fluorosis in two cities with and without artificial fluoridated water, in 1987 and 1997/98. J Dent Res, v. 78, n. 5, p. 985, May 1999. [Abstract A-194]

19. THYLSTRUP, A.; FEJERSKOV, O. Clinical appearance of dental fluorosis in permanent teeth in relation to histologic changes. Community Dent Oral Epidemiol, v. 6, n. 6, p. 315-328, Nov. 1978.

20. VAN DER WEIDJE, G. A.; TIMMERMAN, M. F.; NIJBOER, A. et al. Comparison of different approaches to assess bleeding on probing as an indicator of gingivitis. J Clin Periodontol, v. 21, n. 9, p. 589-594, Oct. 1994.

21. WEINE, S. A construção do paradigma de promoção de saúde - um desafio para as novas gerações. In: ABOPREV. Promoção de saúde bucal. São Paulo : Artes Médicas, 1997. p. 1-28.

22. WhitTle, K. W.; WhiTTLE, J. G. Dental caries in 12-year-old children and the effectiveness of dental services in Salford, UK, in 1960, 1988 and 1977. Br Dent J, v. 184, n. 8, p. 394-396, Apr. 1998.

Recebido para publicação em 16/08/00 Enviado para reformulação em 14/02/01 Aceito para publicação em 27/03/01 